\section{New targets for HIV}

\section{By Kai-Jye Lou, Staff Writer}

The mechanisms by which HIV binds to and fuses with host cells are well documented, but the pathway mediating the intermediate steps between binding and fusion is murky. Now, researchers in France and Italy have teased out a signaling cascade modulated by purines that leads to fusion following HIV-1 binding. ${ }^{1}$

The findings open up a suite of new therapeutic target candidates, as inhibiting any member of the purinergic pathway impaired the replication of both standard and drug-resistant strains of the virus.

Originally, the researchers were studying whether HIV-induced alterations of a host cell's plasma membrane facilitated the process of infection.

"Recent studies had revealed that membrane stress induced by infection or other stressors stimulates ATP release. We decided to determine whether ATP was released during HIV-1 infection and what could be its precise contribution to the early steps of infection," said Jean-Luc Perfettini, a senior scientist and group leader of the apoptosis, cancer and immunity research unit at the Institut National de la Santé et de la Recherche Médicale (INSERM) at Gustave Roussy Institute.

In the process of elucidating the role of ATP

\begin{abstract}
"We discovered that ATP released during the infection process from HIV-1 target cells is indispensable for viral uptake and identified the ATP receptors involved in this process."

- Jean-Luc Perfettini,
\end{abstract}

Institut National de la Santé et de la Recherche Médicale integrase inhibitors and isolates from patients receiving a standard triple-drug cocktail.

Results were published in The Journal of Experimental Medicine. The study was carried out by a consortium of researchers from INSERM, the Centre National de la Recherche Scientifique (CNRS), the National Institute for Infectious Diseases Lazzaro Spallanzani, the Pasteur Institute and universities in France and Italy.

Guido Kroemer, professor in the faculty of medicine at the University Paris Descartes and director of the apoptosis, cancer and immunity unit at INSERM, said the pathway highlights a new set of therapeutic targets for HIV.

"Pannexin 1 is the channel through which ATP is released and can be blocked pharmacologically. ATP can be destroyed by the administration of apyrases, which are ATP-degrading enzymes. P2Y2 is the receptor for ATP and can be inhibited by specific antagonists. PYK2 is a tyrosine kinase and hence druggable," he told SciBX.

"We discovered that ATP released during the infection process from HIV-1 target cells is indispensable for viral uptake and identified the ATP receptors involved in this process," added Perfettini. "Based on our results, we think that the development of topical gels or orally available drugs containing pannexin 1 inhibitors, ATPdegrading enzymes, P2Y2 antagonists or PYK2 inhibitors could constitute effective strategies for preventing or combating HIV-1 infection."

Kroemer and Perfettini were corresponding authors on the paper.

"This study is an elegant detailing of a cascade that is relevant to viral fusion," said Ronald Collman, a professor of medicine at the Perelman School of Medicine at the University of Pennsylvania and co-director release in HIV-1 infection, the researchers identified a purinergic signaling pathway that facilitates intermediate steps between HIV-1 binding and fusion (see Figure 1, "Model of a purinergic signaling pathway in HIV infection").

In human cells expressing both CD4 and CXC chemokine receptor 4 (CXCR4; NPY3R), proteins on the cell surface that HIV-1 uses to infect host cells, exposure to HIV-1 particles stimulated rapid ATP release through the transmembrane channel pannexin 1 (PNX1).

Looking downstream of ATP release, the researchers studied a panel of six purinergic receptors and found that depletion of the ATP-activated purinergic receptor P2Y G protein-coupled 2 (P2RY2; $\mathrm{P} 2 \mathrm{Y} 2)$ caused the strongest decreases in HIV-1 internalization into target cells.

P2Y2 is known to activate protein tyrosine kinase $2 \beta$ (PTK2B; PYK2) through phosphorylation. Indeed, the group found that knockdown of PYK2 reduced HIV-1 internalization and infection compared with no PYK2 knockdown.

Importantly, inhibiting each component of this signaling cascadePNX1, ATP, P2Y2 or PYK2-decreased infection of human cells by standard HIV-1 isolates, isolates resistant to reverse transcriptase and of The Penn Center for AIDS Research. "The data show that there is a lot more to the fusion process than we have come to appreciate and therefore a lot more potential targets in which to intervene."

Collman thinks compounds targeting the signaling pathway might be useful as adjuncts to existing antiretrovirals, such as a viral entry inhibitor, and also could help reduce the likelihood of the virus developing resistance to other agents.

He said it would be valuable to determine whether agents targeting this pathway would have synergy with current drugs that target viral entry, such as Selzentry maraviroc and Fuzeon enfuvirtide.

Selzentry is a CC chemokine receptor 5 (CCR5; CD195) antagonist from Pfizer Inc. Fuzeon is an HIV gp41-targeting viral fusion inhibitor marketed by Trimeris Inc. and Roche.

Franco Lori, founder, president and CEO of Virostatics s.r.l., said some of the targets in the pathway, such as PYK2, will be much more amenable to therapeutic development than others.

"For example, extracellular ATP signaling is a common pathway to many processes, so I would be cautious when developing compounds that go directly after ATP." 


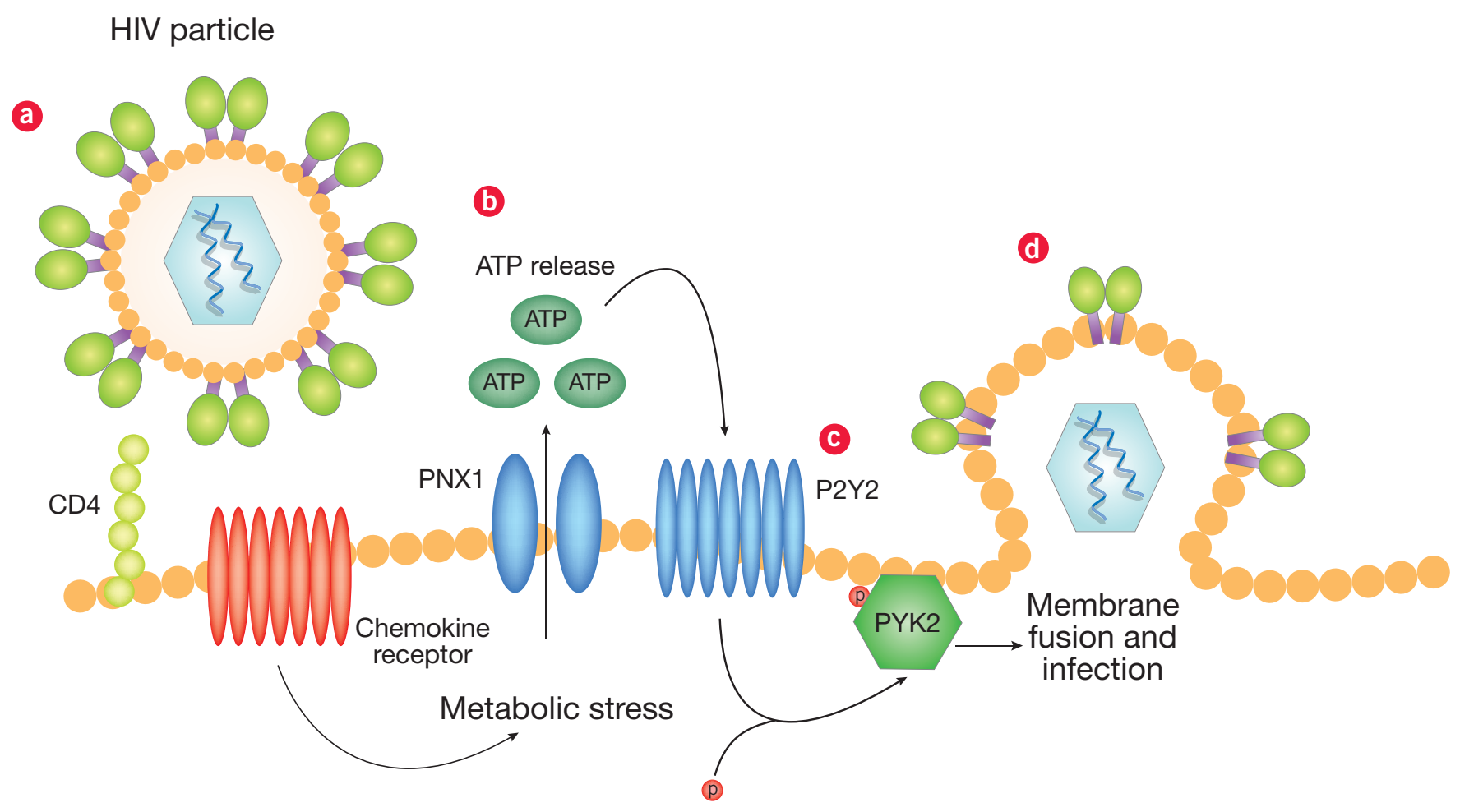

Figure 1. Model of a purinergic signaling pathway in HIV infection. In the current view of HIV fusion and entry, HIV gp120 (green ovals) binds to CD4, which leads to a conformational change in the glycoprotein that exposes HIV gp41 (purple rectangles). This change promotes gp120 binding to a chemokine receptor on the cell-either CXC chemokine receptor 4 (CXCR4; NPY3R) or CC chemokine receptor 5 (CCR5; CD195).

The binding of gp120 to either of these receptors enables the fusion between the viral and cellular membranes, which leads to release of the viral payload into the cell.

Séror et al. have reported that a purinergic signaling cascade mediates the steps between viral binding and fusion and thus have identified a suite of new candidate therapeutic targets for HIV-1.

In their model, binding of the HIV-1 glycoprotein complex to CD4 and the chemokine receptor [a] triggers a metabolic stress response that results in ATP release through pannexin 1 (PNX1) [b]. This ATP activates purinergic receptor P2Y G protein-coupled 2 (P2RY2; P2Y2), which promotes the activation of protein tyrosine kinase $2 \beta$ (PTK2B; PYK2) via phosphorylation (p) [c]. Together, this leads to changes in the properties of the cellular membrane that promote fusion with the viral membrane, thus resulting in infection [d].

Virostatics is developing antiviral hyperactivation-limiting therapeutics, which decrease viral replication and protect the immune system from chronic hyperactivation associated with many viral infections. The company's lead compound is VS411, an enterically coated, fixed-dose combination of dideoxyinosine and hydroxycarbamide that has completed Phase IIa testing for HIV.

\section{The push for potency}

"The study provides the scientific rationale for targeting this pathway, but the translational step now is to take inhibitors against targets in this pathway, such as P2Y2, and measure their activity against a panel of HIV strains to obtain $\mathrm{IC}_{50}$ values," said David Martin, SVP of drug development and regulatory affairs at Tobira Therapeutics Inc.

Tobira's lead compound is cenicriviroc (TBR-652), a dual CCR5 and CCR2 (CD192) antagonist in Phase IIb testing to treat HIV-1.

"The researchers give a clear path for signal transduction, identify four new targets, and use pharmacological inhibitors in the study, which is fantastic," said Fatah Kashanchi, a professor of microbiology and director of research at the National Center for Biodefense and Infectious Diseases at George Mason University.

"But most of the inhibitors they used are general in nature and appear to have $\mathrm{IC}_{50}$ values in the micromolar range. Thus, the focus should be on developing and evaluating next-generation compounds to see whether the data hold and could be improved upon. For the development of HIV drugs, a sub-100 nanomolar $\mathrm{IC}_{50}$ value is usually the unspoken cutoff for a drug candidate to be taken seriously."

They will also need to show that their compound "will block over 99\% of HIV infectivity and replication in cells," added Juan Lama, cofounder, president and CEO at discovery-stage antivirals company RetroVirox Inc.

Collman said additional mechanistic studies could identify more new targets. "The researchers have not yet identified the proximal 


\section{ANALYSIS}

trigger for ATP release through pannexin 1, nor have they defined how the pathway actually modulates viral fusion at the distal side of the signaling cascade," he said.

Kroemer and Perfettini said the group is now working to characterize additional molecular and cellular events required for ATP release and the subsequent signal transduction pathway that stems from ATP's binding to purinergic receptors during viral infections.

Kroemer added that the group is also collaborating with the Institute of Emerging Diseases and Innovative Therapy to develop a primate model that can be used to validate the in vitro results.

INSERM and the Pasteur Institute have filed a patent application covering the findings. The work is available for licensing.

Lou, K.-J. SciBX 4(36); doi:10.1038/scibx.2011.1005

Published online Sept. 15, 2011

\section{REFERENCES}

1. Séror, C. et al. J. Exp. Med.; published online Aug. 22, 2011; doi:10.1084/jem.20101805

Contact: Guido Kroemer, Gustave Roussy Institute, Villejuif, France e-mail: kroemer@orange.fr
Contact: Jean-Luc Perfettini, same affiliation as above e-mail: perfettini@orange.fr

\section{COMPANIES AND INSTITUTIONS MENTIONED}

Centre National de la Recherche Scientifique, Paris, France

George Mason University, Manassas, Va.

Gustave Roussy Institute, Villejuif, France

Institut National de la Santé et de la Recherche Médicale,

Villejuif, France

Institute of Emerging Diseases and Innovative Therapy,

Fontenay-aux-Roses, France

National Institute for Infectious Diseases Lazzaro Spallanzani,

Rome, Italy

Pasteur Institute, Paris, France

The Penn Center for AIDS Research, Philadelphia, Pa.

Perelman School of Medicine at the University of Pennsylvania,

Philadelphia, $\mathrm{Pa}$.

Pfizer Inc. (NYSE:PFE), New York, N.Y.

RetroVirox Inc., San Diego, Calif.

Roche (SIX:ROG; OTCQX:RHHBY), Basel, Switzerland

Tobira Therapeutics Inc., Manalapan, N.J.

Trimeris Inc. (NASDAQ:TRMS), Durham, N.C.

University Paris Descartes, Paris, France

Virostatics s.r.l., Sassari, Italy 